\title{
Airway driving pressure in ARDS patients. Any relationship with lung stress?
}

\author{
Chiumello D., Crimella F., Colombo A., Froio S., Coppola S., Guanziroli M.T. \\ Fondazione IRCCS Ca' Granda - Ospedale Maggiore Policlinico, \\ Dept of Anaesthesiology \& Intensive Care, Milano, Italy
}

\section{Background and Goal of Study}

The lung protective ventilation strategy suggests in ARDS patients the use of low tidal volume associated with moderate to higher levels of PEEP. This strategy should minimize the lung stress and strain and the opening and closing trauma. However in ARDS patients due to the presence of lung disease, the same tidal volume can distribute very differently in the remaining lung volume generating different lung stress (the applied force to the lung) and strain (the magnitude of lung deformation). A possible solution should be to evaluate the airway driving pressure (i.e. the pressure generated for a given tidal volume normalized for the compliance of the respiratory system). Recently Amato et al., hypothesizing that airway pressure driving is an adequate surrogate of the lung strain, showed that airway driving pressure was significantly related to outcome.' However due to the presence of alterations in chest and lung elastance the same inspiratory airway pressure can be generated by different tidal volume. Consequently the transpulmonary driving pressure should better reflects stress. ${ }^{2}$ Aim of this study was to evaluate the relationship between airway and transpulmonary driving pressure in a group of sedated and paralyzed patients at different PEEP level and to evaluate the accuracy of airway driving pressure to estimate the lung stress.

\section{Material and Methods}

91 sedated and paralyzed ARDS patients were enrolled (mean age $6 \mathrm{I} \pm 16$ years, body mass index $27.1 \pm 6.5 \mathrm{Kg} / \mathrm{m}^{2}, \mathrm{PaO}_{2} / \mathrm{FiO}_{2}$ I76 $\pm 6 \mathrm{I}$, PEEP II $.8 \pm 3.2 \mathrm{cmH}_{2} \mathrm{O}$, tidal volume $8.1 \pm 2.6 \mathrm{~mL} / \mathrm{ldeal}$ Body Weight). Airway driving pressure was calculated as the airway pressure changes from PEEP to end-inspiratory plateau pressure. It is equivalent to the ratio between the tidal volume and compliance of respiratory system.' Transpulmonary driving pressure was computed as the difference between airway pressure changes from PEEP to end-inspiratory plateau pressure and esophageal pressure changes from PEEP to endinspiratory plateau pressure. Lung stress was computed as the difference between end-inspiratory airway plateau pressure and esophageal pressure changes from atmospheric pressure to end-inspiratory plateau pressure. A lung stress of $26 \mathrm{cmH}_{2} \mathrm{O}$ was considered the upper limit to minimize the ventilator induced lung injury.

\section{Results and Discussion}

The transpulmonary driving pressure was significantly related to the airway driving pressure $\left(r^{2}=0.79, p<0.000\right.$ I, figure I). However, the transpulmonary driving pressure was better related to the total lung stress compared to airway driving pressure $\left(r^{2}=0.48, p<0.0001 ; r^{2}=0.35, p<0.000\right.$ I figure 2,3$)$. The sensibility and specificity of airway driving pressure to predict a lung stress higher than $26 \mathrm{cmH}_{2} \mathrm{O}$ were 0.66 and 0.92 at 15 $\mathrm{cmH}_{2} \mathrm{O}$, respectively.

\section{Conclusion}

Transpulmonary driving pressure can predict the lung stress better than airway driving pressure, due to the different alteration in lung and chest wall elastance among ARDS patients. However airway driving pressure is simpler, because it does not require the measurement of esophageal pressure by a dedicated balloon, which, for several reasons, is not routinely clinically performed.
Figure I. Regression between airway driving pressure and transpulmonary driving pressure.

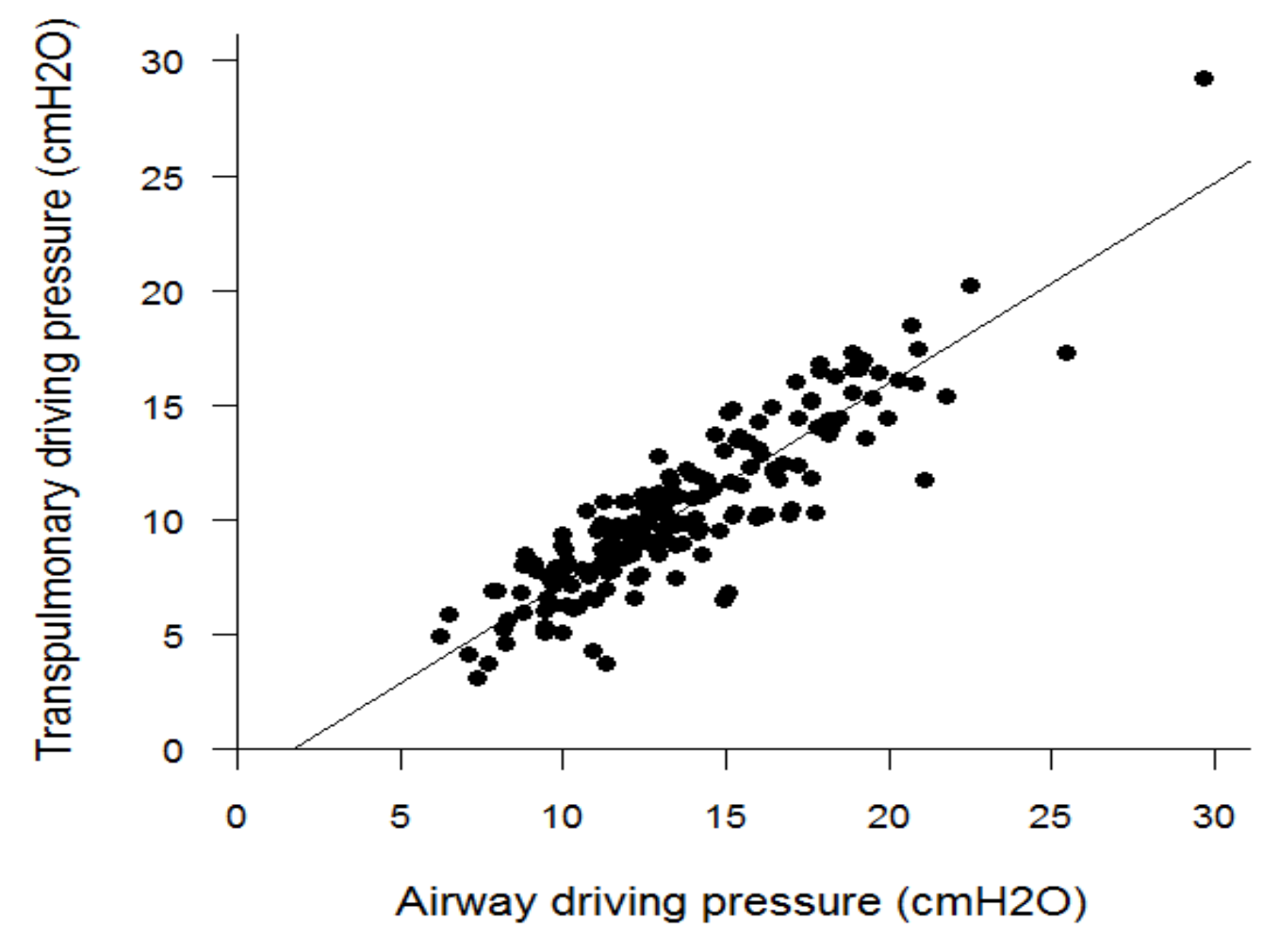

Figure 2. Regression between lung stress and transpulmonary driving pressure.

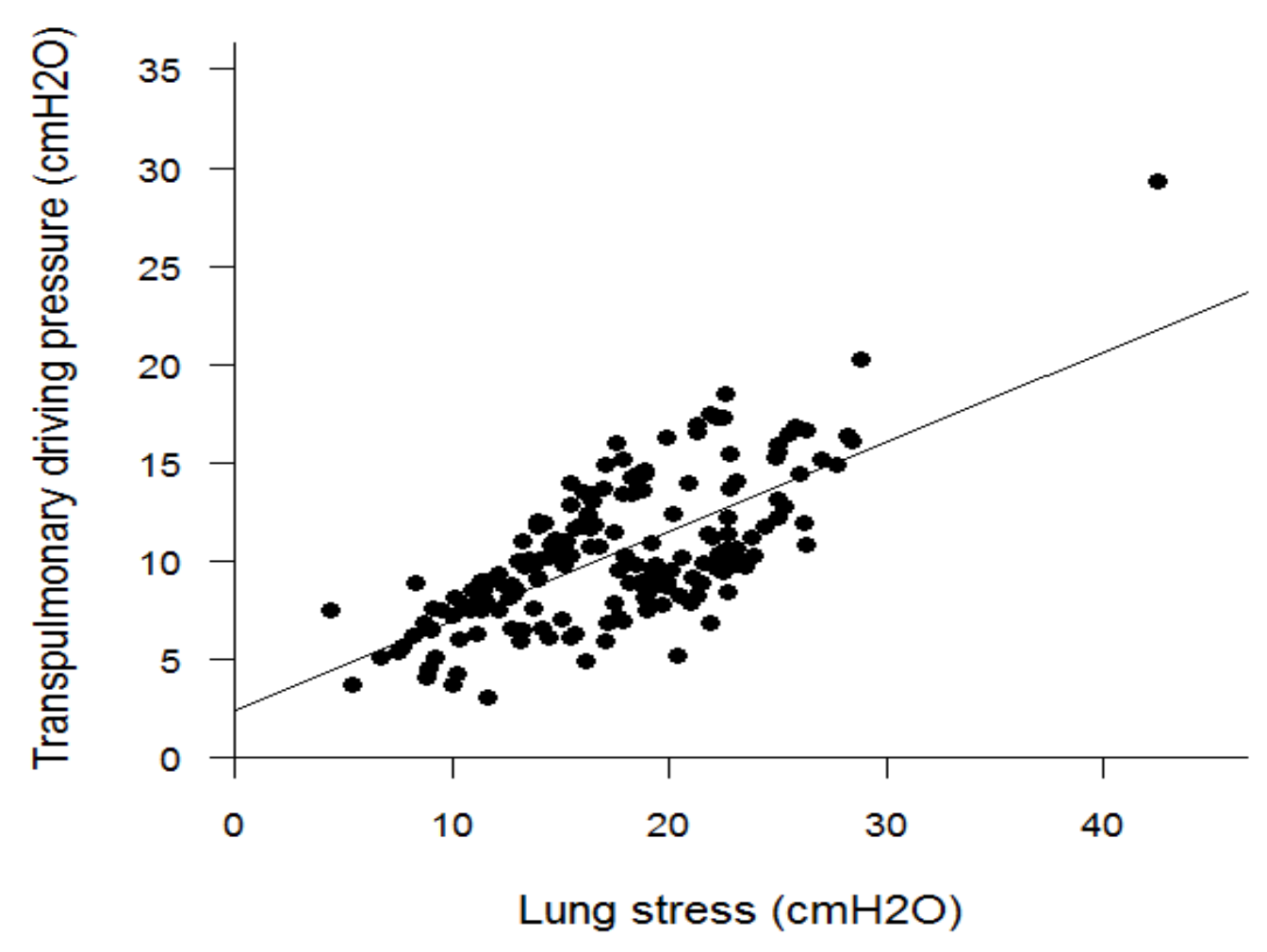

Figure 3. Regression between lung stress and airway driving pressure.

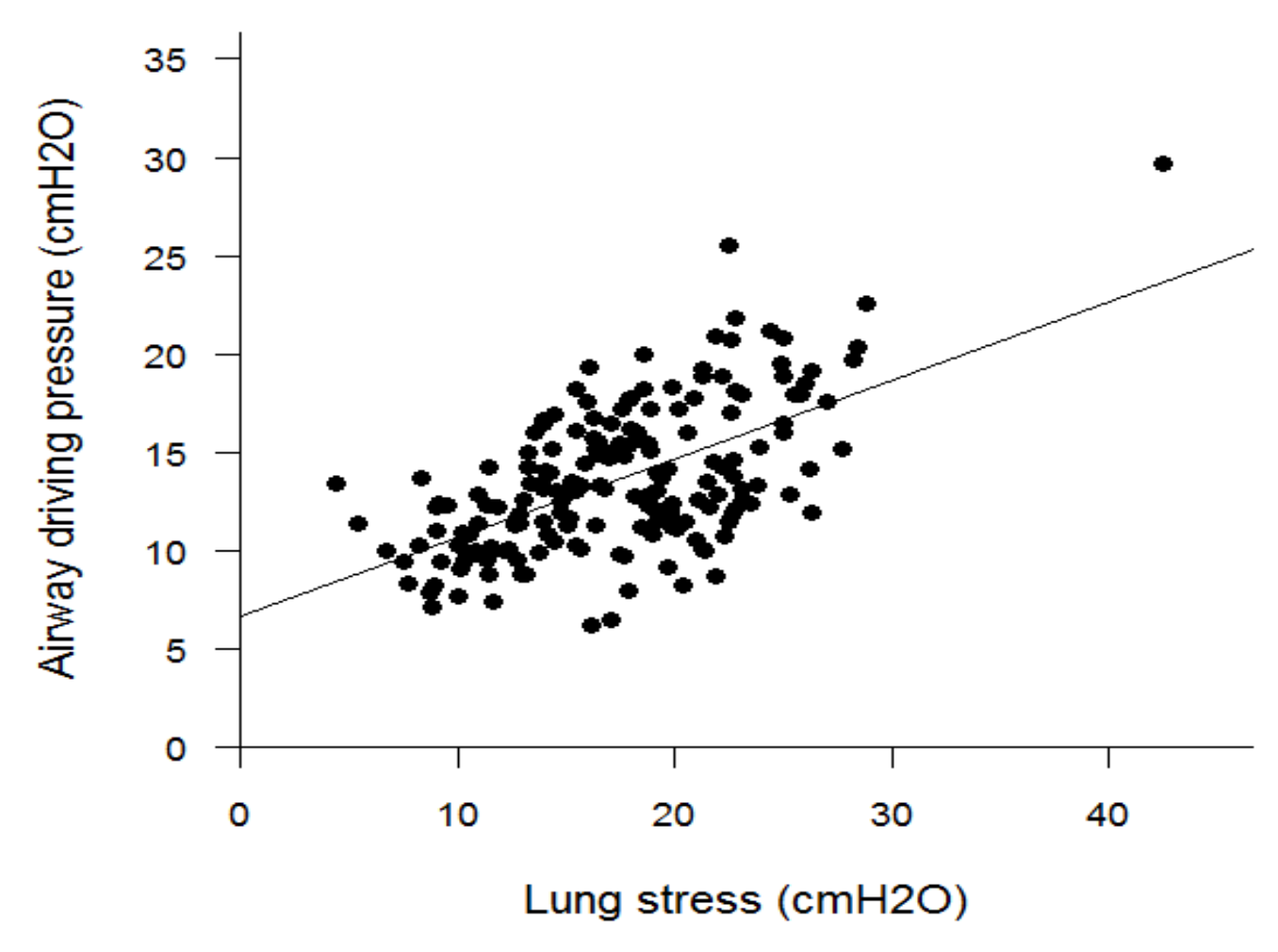

